\title{
TÉCNICAS DE MANEJO COMPORTAMENTAL NÃO FARMACOLÓGICAS NA ODONTOPEDIATRIA
}

\section{BEHAVIORAL MANAGEMENT TECHNIQUES NON-PHARMACOLOGICAL IN PEDIATRIC DENTISTRY}

\author{
Lívia Fernandes Pires da Silva* \\ Nathalia de Carvalho Freire** \\ Rodrigo Silva de Santana*** \\ José Massao Miasato****
}

\begin{abstract}
RESUMO
É habitual, na vivência odontológica, deparar-se com variados tipos de comportamentos indesejados, gerados principalmente pelo medo, ansiedade, birra ou dor, interferindo no atendimento odontológico. Nesses casos, o odontopediatra poderá utilizar técnicas de manejo comportamental não farmacológicas, auxiliando, assim, no tratamento do paciente infantil. Entretanto, para que tais técnicas sejam mais efetivas, é necessário conhecer suas possíveis restrições quanto à faixa etária e perfil de cada criança, de modo a promover e estabelecer segurança e qualidade ao atendimento. Este trabalho tem como objetivo revisar e discutir por meio de literaturas as técnicas de controle comportamental em odontopediatria.
\end{abstract}

Descritores: Odontopediatria • Criança • Controle comportamental • Ansiedade ao tratamento odontológico

\section{A B S TRACT}

It is customary in the dental experience to face different kinds of unwanted behaviors, and these are generated mostly by fear, anxiety, tantrums or pain, thus interfering with dental care. In these cases, the dentist can use behavioral management techniques non-pharmacological, thereby assisting in the treatment of child patient. However in order to such techniques be more effective, it is necessary to know their potential restrictions on the age and profile of each child in order to promote and establish safety and quality service. This work aims to review and discuss through the literatures the behavioral control techniques in pediatric dentistry.

Descriptors: Paediatric dentistry $\bullet$ Child $\bullet$ Behaviour control $\bullet$ Dental anxiety

\footnotetext{
* Graduanda em Odontologia - Unigranrio - Duque de Caxias, RJ. Email: liviapires26@gmail.com

** Graduanda em Odontologia - Unigranrio - Duque de Caxias, RJ. Email: nathaliafreire683@gmail.com

*** Graduando em Odontologia - Unigranrio - Duque de Caxias, RJ. Email: digo_ever@yahoo.com.br

**** Professor da disciplina de Odontopediatria - Unigranrio - Duque de Caxias, RJ. Email: jmassao@gmail.com
} 
SILVA LFP

FREIRE NC

SANTANA RS

MIASATO JM

TÉCNICAS

DE MANEJO

COMPORTAMENTAL

NÃO

FARMACOLÓGICAS

$N A$

ODONTOPEDIATRIA



REV, ODONTOL,

UNIV. CID. SÃO

PAULO

2016; 28(2): $135-42$, MAI - AGO

\section{N T R O D U ÇÃ O}

Embora a área odontológica tenha avançado consideravelmente ao passar dos anos, ainda existe a sensação indesejada relacionada ao medo/ansiedade e expectativa do paciente infantil. No âmbito da odontopediatria, essa sensação pode ter uma proporção maior ou menor, de acordo com cada criança ${ }^{1}$.

O comportamento de uma criança diante do profissional pode ser bastante imprevisível, levando-se em consideração que existem fatores psicológicos relevantes ao tratamento. Entretanto, o odontopediatra deve ter em mente que cada criança possui reações distintas quanto à abordagem odontológica, pois fatores externos podem influenciar negativamente na colaboração do atendimento ${ }^{2}$.

O primeiro contato da criança deverá ser visto de maneira importante e necessária, e que os pais possam prepará-la psicologicamente, a fim de minimizar possíveis anseios com relação ao tratamento ${ }^{3}$. Desse modo, o contato inicial com o odontopediatra não deve ser meramente técnico, mas sim com o intuito de construir uma boa relação entre ambos, para que a criança se sinta única e respeitada ${ }^{4}$.

$\mathrm{O}$ atendimento odontológico infantil requer o gerenciamento comportamental da criança, de forma a viabilizar exames e intervenções relacionados à promoção de saúde. A abordagem deve se adequar à idade, ao gênero, ao estado de saúde geral e aos fatores familiares da criança ${ }^{5}$. O manejo do comportamento terá como auxílio o emprego de técnicas farmacológicas e não farmacológicas, com o propósito de controlar o paciente durante o atendimento.

As técnicas de manejo comportamental podem ser amplamente utilizadas na abordagem da criança no consultório odontológico, possibilitando, assim, a otimização do atendimento. Entretanto, para o sucesso do atendimento, o profissional deve ter conhecimento e embasamento suficiente para discernir uma técnica da outra, elegendo a mais adequada para cada criança ${ }^{3}$.

O estudo em questão lança mão das técnicas não farmacológicas de manejo comportamental como um fator importante no tratamento odontopediátrico. Levando em consideração as diversas maneiras de abordagem infantil.

\section{REVISÃO DE LITERATURA}

O controle de um comportamento indesejado na odontopediatria é quase sempre avaliado através da identificação comportamental e não apenas da faixa etária. Sendo assim, faz-se necessário conhecer os diferentes tipos de comportamento infantil para a correta aplicação das técnicas de controle. Deve-se ressaltar que a comunicação entre a criança e o profissional é um fator que influencia positivamente na colaboração do paciente e na aplicação das técnicas de manejo comportamental não farmacológicas ${ }^{6}$. O comportamento apresentado pelo paciente infantil dará ao profissional a possibilidade de lançar mão de algumas possíveis técnicas de manejo, viabilizando, assim, o controle durante o tratamento odontológico. As técnicas não farmacológicas de manejo comportamental em odontopediatria são utilizadas a fim de gerar segurança e tranquilidade durante o atendimento, sendo as mais utilizadas: comunicação verbal, comunicação não verbal, dizer-mostrar-fazer, controle de voz, reforço positivo, distração, modelo, mão-sobre-a-boca e contenção física ${ }^{5}$.

A comunicação verbal é aplicada de modo a expressar verbalmente os procedimentos, dizendo ao paciente o que será realizado em seu tratamento ${ }^{5}$, enquanto a comunicação não verbal tem como base o contato, a postura, a expressão facial e a linguagem corporal para orientar o comportamento do paciente, reforçando o que foi dito verbalmente ${ }^{1}$.

\section{Dizer-mostrar-fazer}

Essa técnica é uma das mais utilizadas na odontopediatria, pois ela envolve explicações verbais dos procedimentos, utilizando frases/palavras adequadas ao nível de desenvolvimento do paciente (diga); sendo feita em seguida uma demonstração visual e tátil, buscando tranquilizar o paciente infantil (mostre); e a partir da utilização dessa explicação e demonstração, deve-se concluir o procedimento (faça) $)^{5}$. O "dizer-mostrar-fazer" pode ser aplicado 
em conjunto com comunicação verbal e não verbal e reforço. A técnica tem por objetivo ensinar a importância do atendimento odontológico, deixando o paciente à vontade em relação ao atendimento, e assim adaptando-o para se obterem respostas positivas para os procedimentos. É uma técnica indicada para todos os tipos de pacientes ${ }^{1,7}$.

Controle de voz

Trata-se de uma técnica na qual o volume e o tom da voz deverão ser adaptados conforme a necessidade, de modo a influenciarem ou direcionarem o comportamento do paciente infantil ${ }^{8}$, instruindo de forma clara e sucinta e estabelecendo, então, um guia para o comportamento desejado. Essa técnica é primordial no manejo das crianças, pois os pacientes menores normalmente não cedem ao apelo verbal; dessa forma é indicado que o profissional fale baixo continuamente. A entonação é um fator importante, devendo ser utilizada com o intuito de atrair a atenção da criança. A expressão facial do odontopediatra também deve refletir essa atitude de confiança, pois, com a existência de um comportamento perturbador por parte da criança, o controle de voz poderá restabelecer rapidamente uma relação entre dentista e paciente. Assim, a criança obterá a orientação e o profissional terá a cooperação dela. O controle da voz tem o intuito de captar a atenção e a cooperação da criança, podendo, assim, evitar comportamentos negativos do paciente infantil. A técnica é indicada para todos os tipos de pacientes e contraindicada para deficientes auditivos 9 .

Distração

Essa é uma técnica que tem como principal objetivo desviar a atenção da criança para evitar um possível desconforto com algo do qual ela possa vir a ter receio. O dentista deve utilizar procedimentos eficientes para estimular a criança ao tratamento odontológico, pois a tensão psicológica gerada pela situação dentro do consultório pode acarretar ansiedade e medo no paciente. O profissional deve tornar o ambiente favorável ao tratamento, alcançando, desse modo, melhor resultado. Podem ser utilizadas estratégias de manejo como músicas, vídeos e histórias infantis. A música é a mais importante para auxiliar no tratamento odontopediátrico, pois ela pode diminuir o nervosismo e aliviar os sons de alguns aparelhos ${ }^{10,11}$. Podemos também acrescentar métodos que complementem e colaborem para melhor aproximação entre o paciente e o profissional, como conversar com a criança sobre outros assuntos e permitir que utilize algum brinquedo desde que não atrapalhe o procedimento ${ }^{1}$. Essa técnica citada pode ser indicada para qualquer faixa etária infantil, sem contraindicações ${ }^{5}$.

Reforço Positivo

É um processo de motivação do comportamento positivo da criança através de elogios, gestos positivos, expressão facial etc. Essa técnica visa recompensar comportamentos desejados, tendo como principal objetivo o retorno desse bom comportamento ${ }^{9}$. Pacientes infantis podem estar nervosos ou ansiosos e assim não cooperarem ou não seguirem as instruções. Caso eles se sintam ameaçados por algum motivo em particular, isso dificultará a sua cooperação. Em caso de comportamentos negativos, o profissional deve se manter calmo e evitar falar palavras como "Pare", "Não faça", tentando sempre explicar e pedir gentilmente para que a criança se mantenha calma e que realize o que está sendo proposto. Para pacientes infantis abrir a boca é uma grande demonstração de confiança, e elogiá-los repetidamente por seguirem as instruções pode fortalecer essa relação de confiança. Podem ser utilizados reforçadores como lembrancinhas, brinquedos ou até mesmo um simples balão com a luva de procedimento limpa que geralmente é utilizada pelo dentista. O reforço positivo pode ser usado em todos os pacientes ${ }^{1}$.

\section{Modelo}

Essa é uma técnica na qual o clínico utiliza vídeos ou outra criança, que já está condicionada e adequada ao tratamento, servindo de modelo para o paciente que está tendo o primeiro contato com o dentista ou já teve alguma experiência não tão interessante. Dessa forma, é possível ajudá-lo a ter um novo padrão de comportamento, evitando ou reduzindo prováveis negações ou medos prévios que possam existir no paciente, pois a maior parte do
SILVA LFP

FREIRE NC

SANTANA RS

Miasato JM

TÉCNICAS

DE MANEJO

COMPORTAMENTAL

NÃO

FARMACOLÓGICAS

$N A$

ODONTOPEDIATRIA
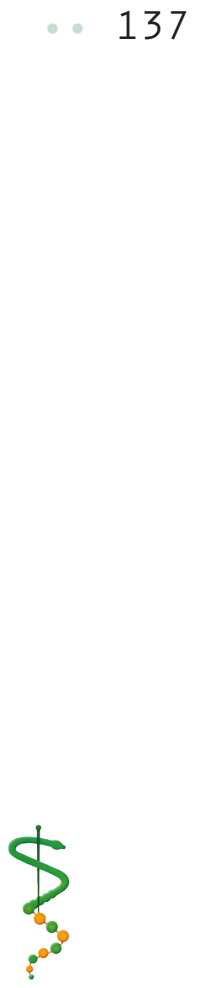

REV. ODONTOL.

UNIV. CID, São

PAULO

2016; 28(2):

$135-42$, MAI - AGO 
SILVA LFP

FREIRE NC

SANTANA RS

MIASATO JM

TÉCNICAS

DE MANEJO

COMPORTAMENTAL

NÃO

FARMACOLÓGICAS

NA

ODONTOPEDIATRIA

$138 \ldots$

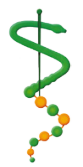

REV, ODONTOL,

UNIV, CID, SÃO

PAULO

$2016 ; 28(2):$

$135-42$, MAI - AGO aprendizado das crianças é baseada em sua observação e imitação de outros ${ }^{10}$. Entretanto, os pacientes observadores devem estar dispostos e excitados com o fato de estarem assistindo outra criança em seu tratamento e o modelo acaba ganhando um status ou prestígio maior, pois ela estará servindo de exemplo positivo para outra criança. Pais, irmãos, colegas e o próprio dentista também podem servir de modelos, porém podem influenciar negativamente caso expressem expectativas negativas para a criança ou, no caso dos pais, estejam muito ansiosos. Nessas situações é melhor que os pais permaneçam fora da sala de atendimento, porque para o paciente observador é interessante ter como modelo uma pessoa com calma e confiança. Na técnica modelo se tem como objetivo reduzir a ansiedade de uma criança com experiência anterior e introduzir uma criança no tratamento odontológico. Ela é um instrumento importante no condicionamento do comportamento de crianças em qualquer idade, se mostrando mais efetiva em crianças abaixo dos 7 anos. Deve-se apenas tomar cuidado em casos de urgência, pois o nível de estresse e ansiedade da criança pode estar elevado e não levar ao o resultado desejado; independente da técnica utilizada é importante para o controle da ansiedade da criança estabelecer uma boa comunicação entre profissional-paciente ${ }^{7}$.

\section{Mão-sobre-a-boca (HOME)}

É uma técnica de manejo físico que tem por objetivo a obtenção da atenção e da colaboração da criança durante o atendimento odontológico, para que esta ouça o que o dentista tem a dizer. Embora seja uma técnica um tanto controversa por conta da aceitação dos responsáveis, possui um bom nível de eficácia quando corretamente aplicada e consentida pelos pais. É uma técnica empregada nos momentos de birra, de choro incontrolável e ataques de ira do paciente infantil, quando for impossível manter um diálogo adequado com a criança, devendo ser empregada juntamente com o controle de voz, buscando estabelecer assim uma comunicação favorável com o paciente e a promoção de um atendimento seguro ${ }^{1}$. O método em questão funciona com o pro- fissional posicionando as suas mãos sobre a boca do paciente infantil, com o intuito de abafar qualquer som e simultaneamente promovendo a aproximação no ouvido da criança buscando uma comunicação favorável, recorrendo a uma entonação adequada. Quando esta não for eficaz, o profissional poderá aplicar a variação da técnica, a "mão-sobre-a-boa com restrições das vias aéreas", que consiste em colocar as mãos sobre a boca do paciente juntamente com o fechamento das vias aéreas superiores por no máximo 15 segundos com os dedos polegar e indicador ${ }^{3}$. Havendo a colaboração da criança no emprego da técnica, as mãos deverão ser retiradas imediatamente e a criança deverá ser elogiada por ter colaborado com o atendimento. A técnica mão-sobre-a-boca e sua variação são indicadas para crianças com grau de entendimento de três anos ou mais, livres de problemas mentais, auditivos e que sejam maduras o suficiente para corresponderem aos comandos do odontopediatra ${ }^{12}$. Entretanto, é contraindicada para crianças incapacitadas, imaturas e que estejam sob o uso de medicações que alterem o nível de raciocínio. É de suma importância que esse método de controle seja explicado detalhadamente para que os pais estejam de acordo com a aplicação da técnica, sendo então formalmente autorizada por eles, buscando assim a otimização do atendimento odontológico. Contenção física

Trata-se de uma técnica que conta com o manejo de restringir fisicamente os movimentos impróprios do paciente infantil na intenção de viabilizar o tratamento odontológico. Essa restrição da liberdade dos movimentos poderá ser aplicada parcialmente ou totalmente, fazendo-se o uso de diversos meios e aparatos como: mãos, cintos, fitas e envoltórios de tecidos $^{9}$. A técnica em questão é uma das últimas opções de escolhas dos odontopediatras, pois não se aplica a todos os tipos de crianças, devendo ser empregada apenas naquelas menores de três anos de idade que não cooperam e possuem um grau mínimo de maturidade, naquelas com algum tipo de deficiência mental que também não colaboram ou naquelas com alguma deficiência física que impossibili- 
te o manuseio, tendo-se em vista o intuito de minimizar possíveis riscos de acidentes durante o atendimento e também proporcionando, desse modo, um atendimento seguro e de qualidade ${ }^{13}$. A utilização desse manejo comportamental deverá ser feito juntamente com o consentimento por escrito detalhado dos pais. Devendo ser explicado a eles o método de escolha para que não enxerguem o uso da técnica como uma forma de punição ou agressão pelo fato de a criança apresentar um comportamento não cooperativo, reduzindo, então, a possível existência de queixas clínicas, problemas éticos e legais ${ }^{5}$. Essa técnica comumente é aplicada em crianças menores em situação de urgência odontológica, dispensando, assim, o uso da técnica de anestesia geral. Não deve ser aplicada em crianças cooperativas, quando não houver a possibilidade de aplicação segura devido a algum problema físico e/ou sistêmico ou quando a criança previamente apresentar uma resposta negativa quanto ao uso de outras técnicas. Lembre-se que é oportuno avaliar o nível de cooperação da criança antes de escolher qualquer que seja a técnica de manejo comportamental.

\section{I SCUSSÃO}

Baseando-se nas literaturas, é oportuno que a avaliação dos variados comportamentos infantis perante o atendimento odontológico anteceda a inserção dos métodos de controle comportamental para que estes não sejam empregados de modo aleatório, pois é necessário identificar o potencial de cooperação e possíveis limitações de cada criança, antes de lançar mão de alguma das variadas técnicas de manejo comportamental.

É importante ressaltar a importância da participação dos pais no consultório odontológico durante o atendimento na primeira infância, pois nessa fase o afastamento entre a criança e seus pais pode gerar angústia ou potencializar o medo, o que impede a cooperação. Entretanto, em pacientes não colaboradores a presença dos pais pode dificultar a capacidade de a criança realizar o que é solicitado; nesses casos é melhor que os pais se retirem da sala de atendimento ${ }^{5}$.

Perante um comportamento coopera- tivo ou não cooperativo, pode-se aplicar a técnica que melhor se adeque, desde que esta vise à segurança e ao conforto no atendimento. Algumas dessas técnicas possuem ampla indicação na odontopediatria, que são as de manejo verbal, enquanto outras possuem suas contraindicações e polêmicas, que são as técnicas de manejo físico.

Em relação à aceitação dos pais, foi visto que, dentre as técnicas de manejo comportamental, as de manejo verbal são as que recebem maior aceitação pelos pais, sendo as de manejo físico mais repudiadas, ao ponto de muitos pais afirmarem que nunca aceitariam esses tipos de técnicas ${ }^{14}$.

Dentre as técnicas de manejo verbal estão a dizer-mostrar-fazer, controle de voz, reforço positivo, distração, modelo, comunicação verbal e não verbal. Tais técnicas visam atrair a atenção da criança, minimizando a ansiedade. Os esclarecimentos, através de explicações verbais e demonstrações, reduzem o fator "desconhecido" que acentua as emoções de medo e ansiedade ${ }^{5}$.

Cada uma dessas técnicas possui seu objetivo principal, tendo as palavras como instrumento principal na sua aplicação. Na dizer-mostrar-fazer é apresentado algo desconhecido para a criança, os instrumentais odontológicos, promovendo a familiarização antes da execução do tratamento. No controle de voz tem-se grande eficácia para interceptar comportamentos impróprios, sendo utilizados comandos súbitos e firmes para interromper qualquer ação negativa que esteja sendo praticada ${ }^{3}$. Por sua vez, a técnica de reforço positivo tem como foco o uso de elogios para a volta de comportamentos desejados, mas também se faz o uso de "lembrancinhas" e expressões faciais positivas, assim como da comunicação não verbal, que além de expressões faciais utiliza a postura e linguagem corporal do profissional ${ }^{9}$.

A técnica de distração é baseada em desviar a atenção do paciente de possíveis procedimentos que possam ser desagradáveis, podendo ser utilizados músicas e vídeos. Em estudos mais antigos não se mostrou eficiente, como no estudo de Stark et al. (1989), mas foi eficaz ao ter sido
SILVA LFP

FREIRE NC

SANTANA RS

MIASATO JM

TÉCNICAS

DE MANEJO

COMPORTAMENTAL

NÃO

FARMACOLÓGICAS

$N A$

ODONTOPEDIATRIA

139

REV. ODONTOL.

Univ, CID. São

PAULO

2016; 28(2):

135-42, MA I - AGO 
SILVA LFP

FREIRE NC

SANTANA RS

MIASATO JM :

TÉCNICAS

DE MANEJO

COMPORTAMENTAL

$N$ ÃO

FARMACOLÓGICAS

$N A$

ODONTOPEDIATRIA

\section{$140 \ldots$}

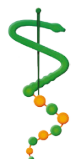

REV, ODONTOL,

UNIV, C I D , SÃO

PAULO

$2016 ; 28(2):$ $135-42$, MAI - AGO usada de forma contingente ao comportamento de colaboração da criança por Ingersoll (1984), citados por Brandenburg e Haydu (2009)2.

Na técnica "modelo" a criança assiste a uma demonstração de um atendimento odontológico através de uma fita de vídeo, teatrinho, ou assiste ao atendimento de outra pessoa. Sendo uma técnica com média aceitação dos pais ${ }^{15}$.

Nas técnicas de manejo físico, conta-se com a contenção física (ativa ou passiva) e com a técnica mão-sobre-a-boca (home ou homar). Essas técnicas também fazem parte do grupo não farmacológico, entretanto são aplicadas de modo a restringir movimentos inapropriados da criança durante o atendimento odontológico, prezando instalar, manter e aumentar bons resultados no tratamento ${ }^{16}$.

A técnica de restrição física, seja ela ativa ou passiva, é um método que deve possuir a autorização por escrito de forma detalhada dos pais, pois, na ausência desse consentimento formalizado, problemas éticos e legais poderão ser gerados. A técnica consiste em conter fisicamente o paciente infantil por meio de mãos, cintos, fitas, lençóis entre outros. É indicada para crianças imaturas, pacientes especiais ou para tratamento de urgências destes, dispensando assim a anestesia geral. $\mathrm{Na}$ maior parte, as crianças sem o uso de contenção física não são cooperadoras e os pais acabam aceitando a utilização dessa técnica, porém ainda é um procedimento com pouca aceitação ${ }^{1}$.

A técnica de mão-sobre-a-boca é utilizada a fim de possibilitar o tratamento odontológico em crianças ausentes de necessidades especiais, que demonstrem comportamentos desafiadores, histéricos, extremamente não cooperativos se impeditivos na realização do tratamento ${ }^{17}$. Segundo Levitas, citado por Ferreira et al. $(2003)^{12}$, nessa técnica deve o cirurgião-dentista colocar a mão sobre a boca da criança, cuidando para não restringir-lhe a respiração (variação da técnica), dizendo em baixo tom, próximo ao ouvido, que, para retirar a mão, a criança deve parar de gritar e escutar, pois somente pretende conversar e olhar seus dentes ${ }^{12}$. Caso o paciente infantil insista em não colaborar, o profissional deverá aplicar a variação da técnica, que seria a mão sobre a boca com restrição das vias aéreas. Embora seja aceita pela American Academy of Pediatric Dentistry (AAPD), a técnica mão-sobre-a-boca mantém-se como a mais controversa dentre as técnicas de limitação usadas por odontopediatras, devendo ser utilizada somente com o consentimento dos pais, que devem estar formalmente de acordo com a possibilidade de sua aplicação. Entretanto, algumas razões apontam para que tal técnica seja aplicada sem a necessidade de autorização dos pais, por ser considerada de difícil explicação por conta de seus efeitos, duração e descrição, podendo causar, desse modo, uma recusa de natureza meramente emocional por parte dos pais $^{12}$. O cirurgião-dentista deve ter pleno domínio de manejo para execução da técnica, para que não a utilize de forma a gerar possíveis lesões no paciente infantil. A aplicação da técnica HOMAR do ponto de vista psicológico, legal e comportamental levanta bastante questionamento, por assumir um papel com característica punitiva. É significativo notar por que HOMAR torna-se eficaz quando HOME não apresenta eficácia: o paciente apresenta-se desconfortável por conta da restrição das vias aéreas, promovendo assim o sucesso da técnica ${ }^{16}$. Quando adequadamente usada, a técnica fará com que a criança aceite o tratamento odontológico, sendo tratada de forma a descobrir que as razões para evitar o tratamento são ineficazes. Sendo assim, o paciente infantil passará a desejar tratamentos futuros e o impacto psicológico logo será positivo ${ }^{12}$.

\section{CONCLUSÃO}

Do exposto, pode-se concluir que, na odontopediatria, existem variadas técnicas à disposição do cirurgião-dentista para abordar os diversos tipos de comportamentos infantis, sendo elas de manejos verbais ou físicos, dentre as quais será utilizada a técnica de eleição que for mais apropriada para empregar na fase de desenvolvimento do paciente infantil.

\section{A GRADECIMENTOS}

- Agradecemos primeiramente a Deus 
SILVA LFP

FREIRE NC

SANTANA RS

MIASATO JM

TÉCNICAS

DE MANEJO

COMPORTAMENTAL

$N A \tilde{O}$

FARMACOLÓGICAS

$N A$

ODONTOPEDIATRIA

142
13. Shitsuka R, Shitsuka C, Moriyama C, Corrêa F, Delfino C, Corrêa M. Desenvolvimento e avaliação da eficiência da estabilização protetora na odontopediatria: um estudo piloto. RFO, PasSo Fundo 2015 jan-abr;20(1):59-63.

14. Machado M, Nagano H, Silva J, Bosco $\mathrm{V}$. Participação dos pais na tomada de decisões no atendimento odontológico de seus filhos. Revista de Odontologia da Universidade Cidade de São Paulo 2009 21(1):38-44.

15. Fúccio F, Ferreira K, Watanabe S, Jorge M, Pordeus I, SM P. Aceitação dos pais em relação às técnicas de manejo do comportamento utilizadas em Odontopediatria. J Bras Odontopediatr Odontol Bebê 2003 6(30):146-51.
16. Sousa C, Barbosa A, Toledo O. Uso de técnicas aversivas de controle de comportamento em odontopediatria. J Bras Odontopediatra Odontol Bebê 2003 6(69):76-82.

17. Lopes Junior C, Carvalho S, Silva R, Peres A. Técnica da mão-sobre-a-boca em odontologia: implicações jurídicas e reflexões bioéticas. Arq ciênc saúde 2005 abr-jun;12(2):97-101.

Recebido em 10/06/2016

Aceito em 08/08/2016
REV, ODONTOL,

UNIV. CID, SÃO

PAULO

2016; 28(2): $135-42$, MAI - AGO 